\title{
Towards Semantic Group Formation
}

\author{
Asma Ounnas \\ Hugh C Davis \\ David E Millard \\ Learning Societies Lab \\ School of Electronics and Computer Science \\ University of Southampton \\ \{ao05r,hcd,dem\}@ecs.soton.ac.uk
}

\begin{abstract}
Many approaches to learning and teaching rely upon students working in groups. Formation of optimal groups can be a time consuming and complex task, particularly when the list of participants is unknown in advance. In this paper we propose an approach to learner group formation, based upon satisfying the constraints of the person forming the groups by reasoning over possibly incomplete semantic data about the potential participants.
\end{abstract}

\section{Introduction}

Collaboration has long been considered an effective approach to learning. Research in many disciplines has shown that learning within groups improves the students' learning experience by enabling peers to learn from each other. There are several forms of collaborative work that allow the students to learn in different ways such as group discussions, peer coaching, problem solving groups, study groups, and social groups. The forms of collaboration differ in purpose, length, complexity of tasks, and degree of formality. For the collaboration to be successful these different forms require different types of groups such as teams, communities of practice (CoPs), intensional networks (INs), and social networks (SNs) [1].

In the learning domain, teachers often have to deal with group formation manually which can sometimes turn into a very complex task; this has led researchers to investigate several techniques for automating this process through the use of computer-supported group formation (CSGF). However, in most existing research, the applications developed model only a limited range of group types. In this paper, we discuss the different approaches to forming the different types of groups mentioned above, the complexity of the formations, and the existing CSGF techniques. We introduce the notion of a system that is capable of constructing different types of groups by semantic reasoning using data about the students and the constraints of group formation given by a teacher.

\section{Group formation (GF)}

In learning, when the need for a collaborative activity is defined, the type of the group that best suits the aim of the collaboration is determined. A specific group formation approach (see section 3) is then chosen to carry out the formation process, which takes place by going through three stages [2]: (1) Initiating the formation, where the instructor or a learner starts the formation. (2) Identifying group members, where the formation initiator chooses who should join which group. This is usually done based on the learners' profiles and the requirements for joining the groups. (3) Negotiating the formation, where the initiator has to ensure the formation satisfies all the members of the group(s) in addition to the formation constraints. In stages (1) and (2), the initiator has to consider two problems:

a. Modeling: In stage (2), the requirements needed to identify the members of each group serve as parameters for the formation. In this context, the initiator needs to identify what parameters need to be modeled. In previous work [1], we discussed which parameters need to be modeled for each group type.

b. Constraint Satisfaction: In stage (3), forming the groups while maximizing the benefits for each student within the group is not an easy task. The formation aims to construct balanced groups in terms of the formation parameters but this approach may conflict with the best interests of individual students. These two factors create the complexity of the group formation.

\section{Group formation approaches}

There are three approaches to constructing groups: 1. Randomly selected groups: are initiated by the instructor who assigns students to groups at random, usually to form temporary informal groups.

2. Self-selecting groups: are initiated by learners who are allowed to choose which group they want to belong to, and can negotiate with whom they want to work with. 
3. Instructor selected groups: are initiated by the instructor. Although teachers can create or direct the creation of students' CoPs and SNs by considering the shared interests and social ties among students, this approach is most popular in task-oriented teams and INs. Table 1 shows the support of the group formation approaches for building different types of groups. The shaded cells highlight the best technique to form each type. Both CoPs and SNs are better formed using selfselection due to their self-organized nature. Hence, for the formation to be effective, the instructor has to provide a degree of self organization within these groups.

\begin{tabular}{|c|c|c|c|}
\hline Groups & Random & Self-selected & Instructor-selected \\
\hline Teams & Partial & Partial & Full \\
\hline CoPs & None & Full & Full \\
\hline INs & None & Partial & Full \\
\hline SNs & None & Full & Full \\
\hline \multicolumn{4}{|c}{ Support: full, partial, none }
\end{tabular}

Table 1: Group formation techniques' support for building the different types of groups.

\begin{tabular}{|l|l|l|l|}
\hline $\begin{array}{l}\text { GF } \\
\text { approach }\end{array}$ & $\begin{array}{l}\text { (1) } \\
\text { Initiator }\end{array}$ & $\begin{array}{l}\text { (2) Identify } \\
\text { members }\end{array}$ & $\begin{array}{l}\text { (3) Negotiate the } \\
\text { formation. }\end{array}$ \\
\hline Random & Instructor & Random & None \\
\hline $\begin{array}{l}\text { Self- } \\
\text { selecting }\end{array}$ & Learner & $\begin{array}{l}\text { Identify } \\
\text { potential } \\
\text { peers }\end{array}$ & $\begin{array}{l}\text { Negotiate with the } \\
\text { identified peers to } \\
\text { join the learner's } \\
\text { group }\end{array}$ \\
\hline $\begin{array}{l}\text { Instructor- } \\
\text { selected }\end{array}$ & Instructor & \multicolumn{2}{|c|}{ Form one group: } \\
\cline { 3 - 4 } & & $\begin{array}{l}\text { Identify } \\
\text { potential } \\
\text { learners }\end{array}$ & $\begin{array}{l}\text { Query on potential } \\
\text { learners, no } \\
\text { negotiation needed }\end{array}$ \\
\cline { 3 - 4 } & & \multicolumn{2}{|c|}{ Group all students: } \\
\cline { 3 - 4 } & $\begin{array}{l}\text { Distribute } \\
\text { students } \\
\text { over groups }\end{array}$ & $\begin{array}{l}\text { Ensure fairness of } \\
\text { formation + } \\
\text { maximize every } \\
\text { student's benefit }\end{array}$ \\
\hline
\end{tabular}

Table 2: Formation process in different group formation approaches

In instructor-selected grouping, the assignment of students to groups involves either the simultaneous distribution of all the students in the class over $n$ groups, where $n$ equals the number of students over the optimal group size; or choosing few students from the entire class to form one group. The last case happens when the collaboration is only needed for a number of students in the class such as using sample students from the whole class population or selecting top students for a specific challenge. The first case is concerned with distributing students evenly to construct balanced groups in terms of the formation constraints, while considering the students' maximum benefits from participating in the groups, in order to ensure active involvement of all students simultaneously, as well as fairly even performances from all the groups (See Table 2). Negotiating the formation in this context is very challenging, and as the number of constraints grows, reaching agreements becomes even more difficult, and the need for CSGF becomes necessary, especially if the number of students involved in the collaboration is large.

\section{Computer-supported group formation}

In [3] Hoppe introduced an intelligent tutoring system that allows the learners to initiate a group formation when they have a problem (a learner-helper group). Based on the learners' models, the system displays a list of all potential peer learners that can help; the learner then selects a helper from the list, and the latter can accept or reject the invitation. Parameters here are based on learning experience in the subject of the collaboration.

The authors in [4] introduced Opportunistic Group Formation (OGF) where the system detects the appropriate situation to start a collaborative learning session and sets up a learning goal for the learner who requires the session, individual learning goals for each learner in the environment, and a goal for the whole group. Based on this, the system negotiates with the agents of all the learners in order to come to an agreement to form a group where each member can obtain some educational benefit. Unfortunately, there is no literature on the developed system or its evaluation.

In similar research [5], the authors introduce a multiagent intelligent system called I-MINDS where the instructor, each student and each group is represented by an agent. The student agent profiles the student and finds compatible students to form the student's "body group". The agents communicate, and form coalitions dynamically in real-time: each student agent bids to join its favorite group based on their previous performance in group work.

Also supporting OGF, in [2], the course author defines at which points in a distributed web based course a collaborative activity should occur. The system forms the groups using knowledge about the collaboration context in real-time such as whether the student has performed this activity before, how often, and how fast.

Redmond [6] introduces a computer program to aid the assignment of students' projects groups using instructorbased approach. The students are grouped, using a greedy algorithm, based on the time slot they prefer to collaborate in, and then allocate the projects to the groups based on the members' preferences in the group.

In terms of formation complexity, we observe that, regarding modeling, most systems only model a fixed set of parameters, which does not allow for the formation of different types of groups. As for constraint satisfaction, most systems use OGF [2], [3], [5], which does ensure satisfaction of the participants in the group through negotiation, but does not discuss the efficiency of the negotiation if all students in the class are grouped simultaneously. Furthermore, OGF is usually more 
beneficial in short term groups. Only in [6] are all the students grouped simultaneously, however, although this research only models one parameter, the evaluation of the system shows that some manual corrections to the results of the formation were needed. Moreover, most existing systems are based on self-selecting approach [3], [4].

\section{Semantic group formation}

With regards to constraint satisfaction, forming balanced groups that satisfy all the participants of the collaboration is a complex task. Here, we introduce the notion of using semantics to reduce the complexity of this problem by employing Semantic Web technologies [7]. In this research we focus on instructor-based group formation as it is the most complex to achieve in CSGF. We aim at building a semantic based system that allows the instructor to automatically form all types of groups.

a. Semantic Modeling: We model a large range of parameters that can be considered for different group formations using the concept of Semantic Web ontologies, which can form a reliable dynamic learner profile [1].

b. Constraint satisfaction: To form the groups, the instructors will be allowed to choose the parameters they want to base the formation on. The formation process itself will involve the executions of a set of rules that represent different formation algorithms. Reasoning on the provided data using the rules to make useful inferences based on the chosen constraints and the students' information provided from their profiles, will allow the students to be assigned to effective groups.

The system will have three main components:

1. The Ontology: This is an extension of friend of a friend (FOAF), an ontology that describes people for building communities and social groupings. We extend FOAF to provide semantic data about the learner for the formation of all types of groups [1]. Each student has an extended foaf file that he or she can update at any time. This allows them to publish data about themselves using a URI, which enables the data to be referred to from any dataset. The files can be updated by the instructor as well, to allow the latter to upload controlled data such as grades, and to check the provenance of the data entered by the students. FAOF also allows the users to define their friends, allowing social connections to be made when using the system to identify CoPs and SNs. As the students can modify their friends' list at any time, the relationships links between them allow the formation to be dynamic, which provides the generated groups with a degree of selforganisation.

2. The Interface: The user will be able to select which parameters they care about for the formation they are initiating. They will be provided by an option that enables them to set constraints on those values and the relationship between those values. The interface will also enable the instructor to rank the importance of these constraints to enable the system to manage compromises based on these priorities. To allow an effective grouping, students are to be encouraged to create meaningful descriptions of themselves. In case they do not provide all required data for a formation, the instructor will be supported by an option that enables the system to look for the data in the web. The strength of semantics in this context relies on the ability to intelligently extract information about the students from web pages and correlate it to the specified constraints in a meaningful way.

3. The group generator: As mentioned before, the group generator will be supported by a set of rules (algorithms) that allows reasoning on the data provided by the learners and the teacher in order to generate effective groups. The system will also enable the instructor to query on the students" data such as "form a group of students that have more than $70 \%$ in the course X", which can be useful in creating sample groups. In general, the system will form a standard semantic technology that allows groups of users to be generated based on a set of constraints and a range of information about themselves.

\section{Conclusion and future work}

In this paper, we discussed the complexity of group formation, and the need for automating it. We then analyzed the limitations of current CSGF applications in terms of their ability to form diverse types of groups, and to effectively satisfy all participants simultaneously while preserving their fairness distribution among the groups. Based on this, we proposed the use of semantics to allow teachers to form different types of groups by reasoning on semantic learners' profiles and the set of constraints that define the formation. In our future work, we examine the use of semantics by implementing the Semantic Web based system that employs these concepts.

\section{References}

[1] Ounnas, A., H.C. Davis, and D.E. Millard, Semantic Modeling for Group formation. In PING workshop at UM07, Corfu, Greece, 2007.

[2] Wessner, M. and H.-R. Pfister, Group formation in computer-supported collaborative learning, Proc. of ACM SIGGROUP, Boulder, Colorado, USA, 2001, pp. 24 - 31.

[3] Hoppe, H.U. Use of multiple student modeling to parametrize group learning, Proc. of AI-ED 95, AACE, Charlottesville, VA, USA, 1995, pp. 234-241.

[4] Inaba, A., T. Supnithi, M. Ikeda, R. Mizoguchi, and J.i. Toyoda. How Can We Form Effective Collaborative Learning Groups? Proc. of ITS, Montréal, Canada, 2000, pp. 282-291.

[5] Soh, L.-K., N. Khandaker, X. Liu, and H. Jiang. A Computer-Supported Cooperative Learning System with Multiagent Intelligence. In AAMAS'06, Hokkaido, Japan, 2006.

[6] Redmond, M.A., A computer program to aid assignment of student project groups, Proc. of ACM SIGCSE, USA, 2001.

[7] Berners-Lee, T., J. Hendler, and O. Lassila, The Semantic Web, in Scientific American. 2001. 